\title{
eJRIEPS
}

Ejournal de la recherche sur l'intervention en éducation physique et sport

$30 \mid 2013$

Varia

Potentialité de la Théorie de l'Action Conjointe en Didactique (TACD) pour l'analyse des situations d'intervention en APS

\section{Chantal Amade-Escot}

\section{(2) OpenEdition}

1 Journals

Édition électronique

URL : http://journals.openedition.org/ejrieps/2466

DOI : 10.4000/ejrieps.2466

ISSN : 2105-0821

Éditeur

ELLIADD

Référence électronique

Chantal Amade-Escot, « Potentialité de la Théorie de l'Action Conjointe en Didactique (TACD) pour l'analyse des situations d'intervention en APS », eJRIEPS [En ligne], 30 | 2013, mis en ligne le 01 juillet 2013, consulté le 24 septembre 2020. URL : http://journals.openedition.org/ejrieps/2466 ; DOI : https://doi.org/10.4000/ejrieps.2466

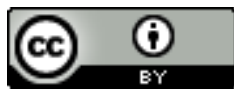

La revue eJRIEPS est mise à disposition selon les termes de la Creative Commons Attribution 4.0 International License. 
eJRIEPS 30 juillet 2013

Potentialité de la Théorie de l'Action Conjointe en Didactique (TACD) pour l'analyse des situations d'intervention en APS

Chantal Amade-Escot

Université Toulouse 2 - Le Mirail, UMR EFTS, MA 122

Depuis les premiers travaux en didactique des Activités Physiques Sportives et Artistiques (APSA) et de l'éducation physique et sportive (EPS), les chercheurs se sont attachés à décrire et comprendre l'activité des acteurs en contexte, qu'ils soient enseignants, entraîneurs ou intervenants dans le monde sportif ou éducatif. Cette contribution aux 6 ème "Journées d'Etude Alain Durey" (JAD) se situe dans le prolongement des premiers travaux menés à l'INRP par Jacqueline Marsenach et Robert Mérand. Les recherches de ces pionniers, irrigués des débats menés avec Daniel Bouthier et Alain Durey au sein du DEA «Enseignement et Diffusion des Sciences et des Techniques» (ENS Cachan, Université Paris $\mathrm{XI}, \mathrm{INRP}$ ) ont constitué le creuset d'un programme de recherches descriptives et compréhensives qui sont aujourd'hui poursuivies sous couvert du cadre théorique de l'action conjointe en didactique (TACD). Les recherches en intelligibilité conduites dans ce cadre théorique et selon les méthodologies d'observation «du " didactique ordinaire qui y sont associées, visent à rendre compte du fonctionnement des systèmes didactiques classiquement définis comme système ternaire d'interactions (Brousseau, 1998). Pour ce faire, elles s'attachent, entre-autre, à documenter à partir d'analyses in situ, l'intentionnalité et les significations accordées par les acteurs aux savoirs mis à l'étude et les manières dont ces derniers agissent et co-agissent au fil de l'évolution de la relation didactique. II ressort de ces prémices que " l'analyse de l'activité en contexte ", thème de ces $6^{\text {ème }} \mathrm{JAD}$, est au cœur des recherches menées. Soulignons par ailleurs leur caractère comparatiste qui amène les didacticiens s'inscrivant dans ce courant, à étudier d'autres systèmes didactiques que ceux relevant de l'enseignement scolaire de l'EPS. Ce programme de recherche sur l'intervention en sport considère avec d'autres (Musard, Loquet et Carlier, 2010), que l'activité humaine se déploie toujours dans une culture et dans des collectifs qui lui donnent sens. Ainsi la «pratique »- même la plus individuelle - est toujours tissée dans des rapports intersubjectifs et par rapport à des 


\section{eJRIEPS 30 juillet 2013}

œuvres. Elle ne prend sens et épaisseur que dans le cadre de transactions impliquant divers acteurs, d'où l'idée « d'action conjointe ».

Avant d'indiquer, comme y invite la thématique centrale de ces journées d'étude, quelques liens possibles entre les recherches développées dans ce programme et la question plus générale de la formation des intervenants en sport, présentons en quelques mots la TACD et son émergence au sein de l'approche comparatiste en didactique telle qu'elle est développée depuis une dizaine d'années.

\section{Bref aperçu de la théorie de l'action conjointe en didactique}

La notion d'action conjointe en didactique apparaît dans les années 80 . On en trouve l'origine dans la thèse de Maria-Luisa Schubauer-Leoni (1986) qui montre la nécessité empirique de prendre en considération à la fois les actions des élèves et des enseignants pour accéder à la compréhension des phénomènes didactiques. Cette option théorique est aussi celle des premières recherches en didactique de l'EPS, dont l'originalité est d'avoir mis en évidence que l'enseignant n'enseigne, à proprement parler, que lorsqu'il régule l'activité des élèves aux prises avec des situations d'apprentissage (Marsenach et Mérand, 1987; Marsenach, 1989). C'est dans la lignée de ces différents travaux qu'émerge, au tournant des années 2000, la TACD alors que se profile un mouvement comparatiste (Mercier, Schubauer-Leoni et Sensevy, 2002). Le postulat central est que les phénomènes transpositifs sont le résultat d'une co-activité entre professeur et élèves relativement à des enjeux de savoirs, d'où l'expression « théorie de l'action conjointe en didactique ». D'un point de vue formel, ce cadre théorique est structuré autour de quelques positionnements théoriques fondamentaux auxquels sont associés des descripteurs permettant l'analyse des corpus :

- S'inscrivant dans les perspectives épistémologiques contemporaines des sciences humaines et sociales, il fait sien les postulats actionnel, culturaliste et non dualiste de ces courants de pensée. Pour résumer, la TACD emprunte ses principaux concepts aux théorisations didactiques antérieures, en particulier celui de «transposition didactique » et son actualisation in situ appréhendée sous couvert de la dialectique "contrat/milieu didactiques". De ce point de vue la TACD marque un «tournant actionnel » dans l'analyse des phénomènes transpositifs et la prise en compte des sujets impliqués dans les transactions qui y sont associées. La fonction du modèle théorique est de rendre intelligible la dynamique interactionnelle (Blumer, 1969 ; Goffman, 1967) présidant aux processus didactiques. Partant de l'idée que l'action didactique conjointe est « située ", le 


\section{eJRIEPS 30 juillet 2013}

modèle développe une approche pragmatiste (Mead, 2006) tout en s'inscrivant dans la perspective historico-culturelle de l'interactionnisme social (Bronckart, 2005).

- La TACD articule plusieurs outils analytiques, qui sont des descripteurs de l'action conjointe. Leur fonction est de rendre compte de la manière dont, au fil du temps didactique, les inter-actants co-construisent un milieu à la fois, matériel, symbolique et social à partir duquel s'élabore une référence. Car « toute situation d'enseignement est l'occasion de donner vie à des pratiques de savoir en référence à des pratiques sociohistoriquement cristallisées en activités. Le processus d'enseignement est dès lors censé recréer certaines conditions à même de permettre l'émergence d'« expériences " jugées compatibles avec les pratiques de références qui sont à l'œuvre dans la culture humaine » (Schubauer-Leoni, Leutenegger, Ligozat, Fluckiger, 2007, p. 53). Ces descripteurs sont de deux ordres : ceux qui rendent compte de l'action du professeur (définir, dévoluer, réguler, institutionnaliser) ; ceux qui marquent les dynamiques évolutives (les genèses) du milieu, du temps didactique (avancée ou stagnation des savoirs au fil des transactions) ainsi que celle des places et des responsabilités de chacun dans ces processus interdépendants (pour un développement voir Sensevy, 2007).

\section{Indications méthodologiques}

Le tournant pragmatiste et actionnel qui caractérise la TACD marque le refus d'un point de vue substantialiste et mentaliste sur les savoirs et valorise une analyse ascendante de la transposition didactique articulant délibérément les dimensions interactionnelle et épistémique des situations observées. Pour ce faire, le protocole méthodologique combine des entretiens et des observations in situ. L'analyse épistémologique s'intéresse aux savoirs en jeu dans les dispositifs d'enseignement et d'apprentissage et à leur évolution au fil des interactions à partir de descriptions produites par le chercheur. II s'agit de confronter les possibles d'un dispositif donné, possibles mis en évidence par le biais d'une analyse a priori et les effets observés du dispositif tel que réalisé (analyse a posteriori). L'analyse a priori consiste à déterminer quels sont les enjeux de savoirs mis à l'étude. Sa fonction est épistémique et exploratoire. II s'agit d'anticiper l'univers des possibles et d'établir en quoi la tâche peut constituer (ou non), sous certaines conditions, un milieu favorable aux savoirs visés (Amade-Escot, 2007). L'analyse interactionnelle vise à rendre compte du processus d'actualisation du milieu et du contrat au fil des transactions. L'évolution du système didactique est documentée à partir des descripteurs de l'action professorale (définir, dévoluer, réguler, institutionnaliser) et du triplet des genèses. Cette analyse, 


\section{eJRIEPS 30 juillet 2013}

menée par le chercheur, est triangulée avec un autre ensemble de données visant à prendre en considération le point de vue des acteurs. II s'agit de suspendre les catégories théoriques de l'analyse du chercheur pour se rendre sur le terrain de l'autre afin d'accéder au sens qu'il attribue à son action à partir d'un ensemble d'entretiens (pour un développement, voir Leutenegger, 2009).

C'est dans la mise en tension de ces analyses que s'élabore l'interprétation. Elle renvoie à la nécessité - dès lors que l'on veut rendre compte de l'action - de distinguer pour mieux les articuler ce qui relève du langage du modèle et de la sémantique naturelle de l'action (Ricœur, 1977). II s'agit donc de produire une description de l'action qui puisse rendre compte à la fois du sens pour le sujet (sémantique naturelle de l'action) et de(s) signe(s) pour le chercheur (logique du modèle). C'est pourquoi, le processus interprétatif croise deux points du vue celui extrinsèque du chercheur et celui, intrinsèque à l'acteur (BrièreGuenoun et Amade-Escot, 2010). Ce croisement a pour but de ne pas dilater démesurément le poids accordé à la capacité interprétative du sujet, tout comme il permet de ne pas attribuer à celui que l'on observe, le sens donné par celui qui est en position d'observateur.

\section{En quoi la TACD a-t-elle une pertinence pour discuter des rapports entre recherche et formation?}

Globalement on peut considérer que la formation didactique des intervenants en sport et en EPS a été principalement construite sur l'idée - fort pertinente par ailleurs - d'une transformation des rapports aux "savoirs à enseigner " des intervenants. Ce postulat, plus ou moins implicite, s'appuie sur les constats établis par nombre de recherches de la nécessité de renouveler les savoirs ou les conceptions des intervenants à propos des contenus de leur intervention. Mais il reste à s'interroger sur les modalités à partir desquelles le changement escompté est envisagé. Les rapports entre recherche didactique et formation des enseignants d'EPS ou des intervenants en sport ont été souvent pensés à partir de dispositifs ou de postures visant à assurer un contrôle épistémologique sur les savoirs ou les formes de pratiques retenus pour l'intervention. De nouvelles propositions de contenus ont ainsi fait l'objet d'expérimentation et ont été validées par diverses formes de recherche : recherche-actions (proposant des innovations contrôlées sur des ensembles d'objectifs, de contenus et de démarches), essaisévaluation de prototypes d'enseignement ou d'entraînement, ingénieries didactiques, etc. Leurs visées stratégiques concourent à un projet plus vaste de re-historicisation et de resocialisation des contenus de l'intervention, dont on présuppose qu'il est à-même de 


\section{eJRIEPS 30 juillet 2013}

réduire les difficultés rencontrées par les intervenants. Du point de vue de l'articulation « recherche » et «formation » didactiques, on se situe là dans une approche descendante des phénomènes transpositifs qui valorise la dimension épistémique de la formation selon une conception de la circulation des savoirs entre la recherche et la formation qui « oublie » leur nécessaire re-location dans l'action (voir par exemple sur ce dernier point, les recherches en formation didactique des enseignants d'EPS menées par Dugal, 2008). L'approche ascendante sous-jacente à la TACD suggère de nouvelles voies pour penser l'articulation entre recherche didactique et formation des intervenants. Elles ont été ouvertes par une inflexion du travail scientifique en didactiques des disciplines apparue au tournant des années 2000 autour de l'analyse des pratiques des intervenants. II convient aussi de souligner les apports, dans le domaine de l'entraînement, des travaux développés sous couvert de la didactique professionnelle et de la technologie des APSA (Bouthier, 2008). Pour sa part, c'est en déclarant que le «lieu d'investigation des phénomènes de transposition didactique [ne se situe] ni (que) dans les savoirs ni (que) dans les sujets - enseignants et apprenants - mais dans leur action conjointe » (Schubauer-Leoni et Leutenegger, 2005, p. 408) que la TACD reconsidère fondamentalement les liens entre recherche et formation didactiques. Le sens que prennent les savoirs pour les sujets (dans les espaces didactiques scolaires ou non scolaires) est fortement lié aux modalités de leur co-production en situation au regard des pratiques culturelles qui les légitiment.

En pointant que les savoirs sont co-construits, en mettant en évidence la dynamique contractuelle différentielle de cette construction, en se rendant sensible enfin, aux processus sémiotiques sous-jacents, la TACD propose de (re)penser la formation des intervenants à partir de l'analyse des pratiques conjointes des acteurs de la relation didactique. Pour autant, cette entrée par l'observation de l'action conjointe ne laisse pas de côté la dimension épistémologique d'analyse des savoirs ; au contraire, elle la valorise dans la mesure où c'est à la lumière de cette dernière qu'elle peut dire quelque chose de la dimension interactionnelle des processus observés. Ce parti-pris impose de réexaminer le statut de l'expérience didactique des intervenants au fil du temps. Il a aussi pour conséquence un changement des rapports entre recherches didactiques et formation des intervenants. C'est donc bien dans le cadre d'analyses didactiques de la pratique que l'on peut penser les relations entre recherche(s) et formation à l'intervention, mais à condition de ne jamais perdre de vue les dimensions historiques, culturelles et épistémiques des contenus de cette intervention. 


\section{Références bibliographiques}

Amade-Escot, C. (2007). Le didactique. Paris : Editions Revue E.P.S.

Bouthier, D. (2008). Technologie des APSA : évolution des recherches et de leur place dans le cursus STAPS. Revue eJRIEPS, 15, 44-59. http://www.fcomte.iufm.fr/ejrieps/ejournal15/Bouthier\%20eJ\%2015.pdf

Brière-Guenoun, F. \& Amade-Escot C. (2010). Analyse in situ des savoirs mobilisés par un professeur d'éducation physique et sportive dans l'interaction didactique. Revue Suisse des Sciences de l'Education, 32(2), 311-333.

Bronckart, J.P. (2005). Une introduction aux théories de l'action. Carnet des sciences de l'éducation. Genève : Faculté de psychologie et des sciences de l'éducation.

Brousseau, G. (1998). Théorie des situations didactiques. Textes rassemblés et préparés par Balacheff, Cooper, Sutherland, Warfield, Grenoble : la pensée sauvage

Blumer, H. (1969). Symbolic Interactionism. Perspective and Method. University of California Press: Berkeley, Los Angeles, London.

Dugal J-P. (2008). Le conseil en formation initiale des enseignants. Intérêt et fonction des savoirs didactiques pour le tutorat des professeurs stagiaires en EPS. eJRIEPS, 14 , 7-21.

http://www.fcomte.iufm.fr/CD RENTREE 2007/rentree/RECHERCHE/ejrieps/ejou rnal 14/sommaire.htm

Goffman, E. (1967). Interaction ritual. New-York: Doubleday

Leutenegger, F. (2009). Le temps d'instruire. Approche clinique et expérimentale du didactique ordinaire en mathématique. Berne : Peter Lang

Marsenach, J. (1989). Les pratiques des enseignants d'EPS dans les collèges. Revue française de pédagogie, 89, 7-10.

Marsenach, J., Mérand, R. (1987). L'évaluation formative en EPS dans les collèges. Rapport scientifique. N2. Paris. INRP.

Mead, G.H (2006). L'esprit, le soi et la société. Paris : PUF, traduction de D. Cefaï et L Quéré.

Mercier, A., Schubauer-Leoni, M.L., et Sensevy. G. (Eds. 2002) Vers une didactique comparée. Revue Française de Pédagogie, 141.

Musard, M., Loquet M. et G. Carlier (Eds) (2010). Sciences de l'intervention en EPS et en sport. Résultats de recherche et fondements théoriques. Paris: Aris \& Editions de la revue EP.S. 


\section{eJRIEPS 30 juillet 2013}

Ricœur, P. (1977). La sémantique de l'action. Paris : CNRS

Schubauer-Leoni, M.L. (1986). Maître-élève-savoir : Analyse psychosociale du jeu et des enjeux de la relation didactique. Thèse de doctorat en Sciences de l'Éducation Université de Genève.

Schubauer-Leoni, M.-L. \& Leutenegger, F. (2005). Une relecture des phénomènes transpositifs à la lumière de la didactique comparée. Revue Suisse des sciences de l'éducation, 27(3), 407-429.

Schubauer-Leoni, M.L., Leutenegger, F., Ligozat, F., et Fluckiger, A. (2007). Un modèle de l'action conjointe professeur-élèves: les phénomènes didactiques qu'il peut/doit traiter. In G.Sensevy, et A. Mercier, (Eds.). Agir ensemble. L'action didactique conjointe du professeur et des élèves (pp. 51-91). Rennes : Presses Universitaires.

Sensevy, G. (2007) Des catégories pour décrire et comprendre l'action didactique. In G. Sensevy \& A. Mercier (Eds.). Agir ensemble. L'action didactique conjointe du professeur et des élèves, (pp. 13-49). Rennes : Presses Universitaires. 